\title{
Cilia Alterations in Models of Polycystic Kidney Disease
}

\author{
V. H. Gattone II*, C. Miller*, B. Blazer-Yost** and R. L. Bacallao* \\ *Indiana University School of Medicine, Depts. of Anatomy \& Cell Biology / Medicine \\ **Purdue School of Science, Dept of Biology, IUPUI, Indianapolis, IN
}

Polycystic Kidney Diseases (PKD) are a group of conditions associated with the development of fluid-filled, epithelia-lined, cysts in kidney. Aside from classical forms of PKD caused by mutation in PKD1, PKD2 and PKHD1 genes, other inherited renal cystic conditions include: Nephronophthisis-NPH, Bardet Biedl Syndrome-BBS, Meckel Syndrome-MKS and Oro-facial-digital Syndrome that encompass 20+ additional gene loci. Most, if not all, of the protein products of genes for these conditions are found in cilia, basal bodies and/or centrosomes [1]. Since cilia are associated with many different extrarenal cell types as well, these conditions are typically associated with pathology in additional organs (i.e. liver, brain, pancreas, eyes, ears, etc). As such, these renal "ciliopathies" are life-threatening diseases which can affect fetuses, infants, adolescents and adults depending on which gene is mutated and the nature of the mutation.

While the basic structure of cilia may appear to be preserved in these renal ciliopathies (Figures $1 \& 2)$, the function of the cilia may be compromised. There is a constant transport of proteins out to the tip and other proteins back to the cell. Mutations in the genes for these intraflagellar transport proteins (IFT) like IFT88 (aka Polaris or orpk) or KIF3a limit or prevent cilia formation and lead to the development of renal cysts $[2,3]$. However, in other renal cystic ciliopathies, cilia may form, but exhibit variability in length $[4,5]$ (Figure $3 \& 4$ ) they may not function properly. Renal cilia appear to function primarily as mechanoreceptors while cilia in olfactory cells are chemosensors. Retinal rod cell cilia are critical light sensors (Figure 6\&7) while vestibular and cochlear hair cells sense movement (Figure 5).

Renal cilia movement elicits a calcium transit that is absent when a PKD gene is mutated [6]. However, there are a number of signaling pathways that are involved in cyst formation and progressive enlargement that appear to be downstream of the cilia abnormality. While mutations in various genes (PKD, NPH, BBS or MKS) cause renal cysts, they all appear to use similar signaling pathways in disease progression (i.e. G protein receptors-cAMP, mTOR pathway, growth factor receptor kinases, etc). While the connection of some pathways to the cilia is tenuous, targeting these similar pathways have limited the renal pathology in animal models and are now being pursued in human Clinical Trials [7].

\section{References}

1. B.K. Yoder, J Am Soc Nephrol. 18 (2007) 1381-1388.

2. J.M. Lehman et al., Dev Dyn. 237 (2008) 1960-1971.

3. F. Lin et al., Proc Natl Acad Sci U S A. 100 (2003) 5286-5291.

4. M.N. Muchatuta et al., Exp Biol Med (Maywood). 234 (2009) 17-27.

5. R. Tammachote et al., Hum Mol Genet. 18 (2009) 3311-3323.

6. S.M. Nauli et al., J Am Soc Nephrol. 17 (2006) 1015-1025.

7. V.E. Torres. Adv Chronic Kidney Dis. 17 (2010) 190-204.

8. Partial support provided by NIH R01 DK068581(VHG) and a EM Core grant from the PKD Foundation (VHG). 

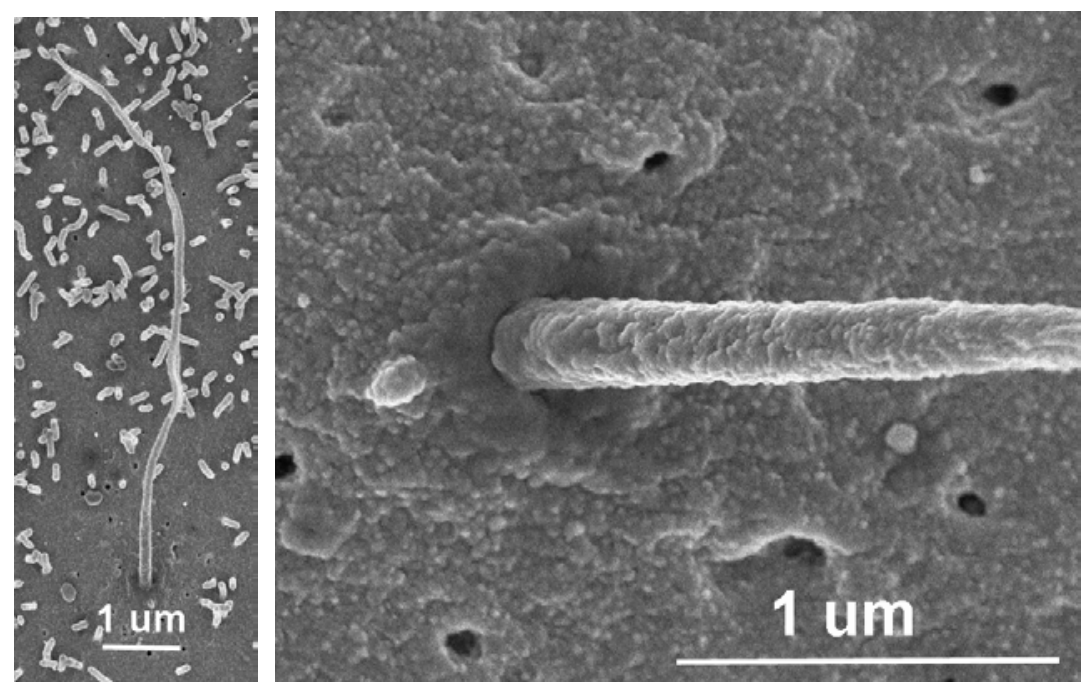

Figures $1 \& 2$. SEM of cultured autosomal dominant PKD renal epithelia demonstrating a cilia (left) and its cellular origin (right). Note the slight depression from which the cilia arises. Note the microvilli (left image) and endocytotic pits (right image) on the apical surface of the cell.
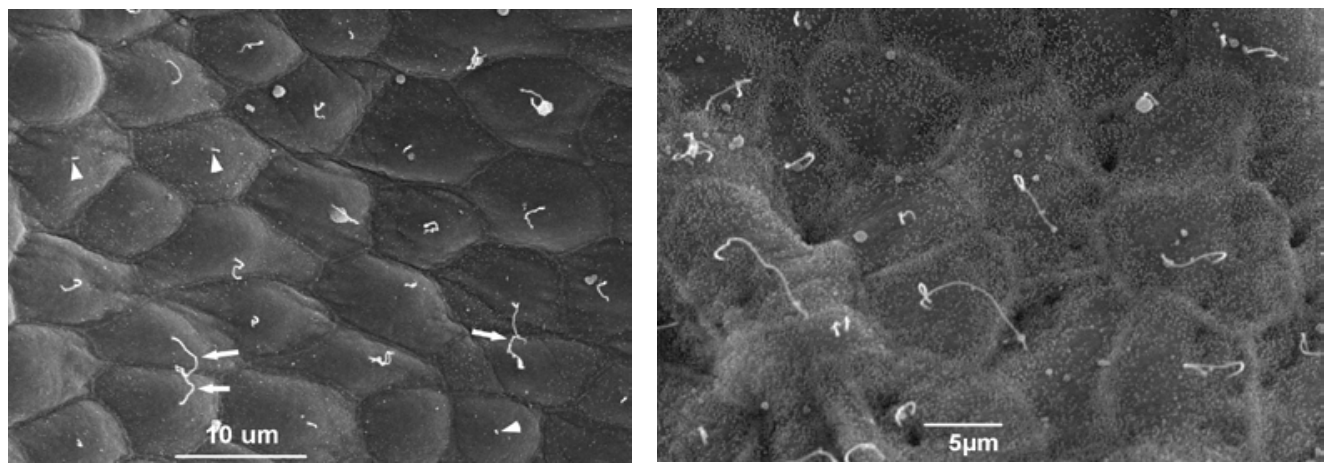

Figures $3 \& 4$. SEM of renal cyst from Balb/c-cpk/cpk and liver cyst from $c p k /+$ mice. Note the variability in cilia length in renal (arrowhead versus arrows, above left) and in liver (above right) cystic epithelia.
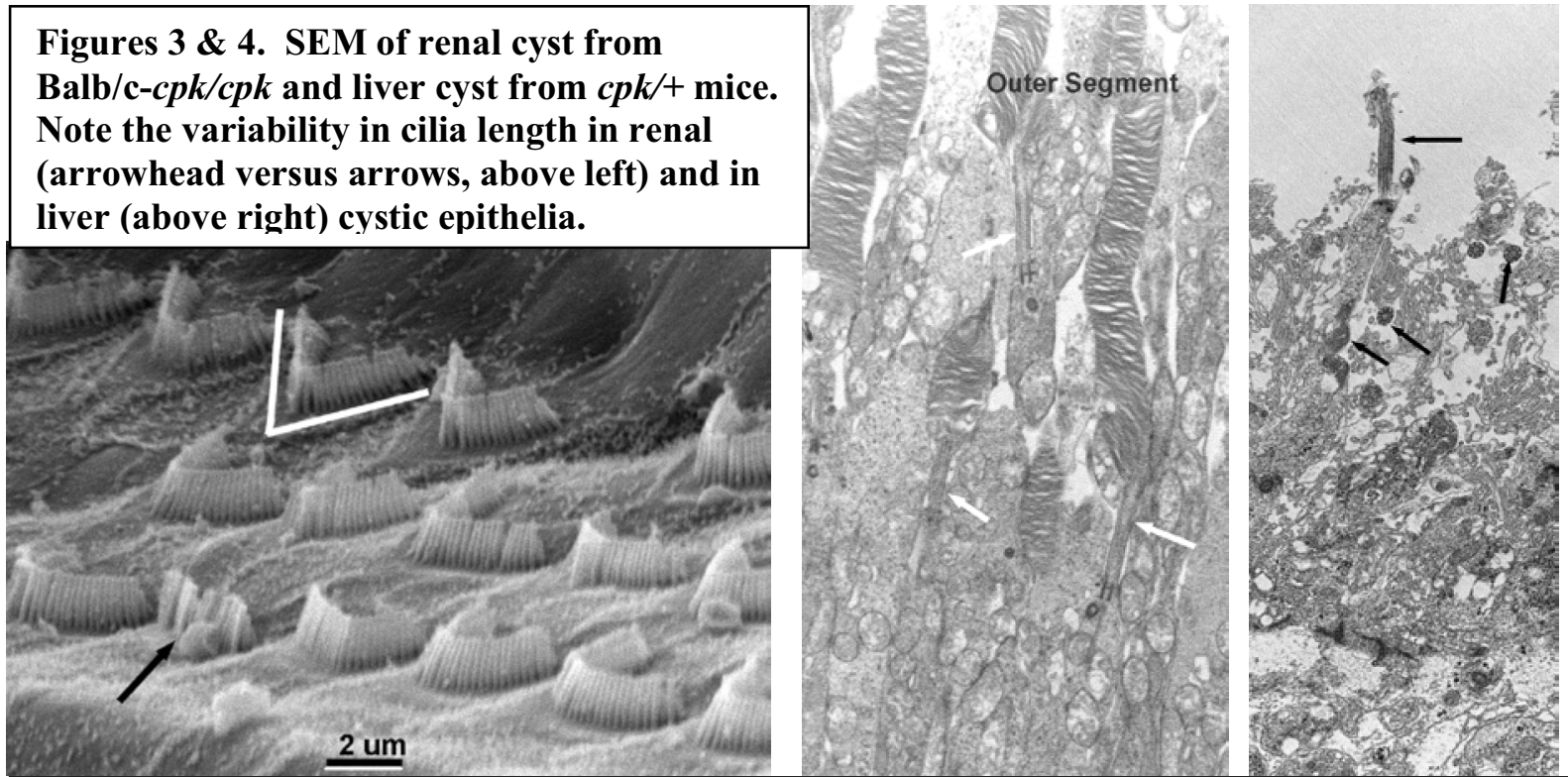

Figures 5, 6 \& 7. SEM of cochlear hair cells from $w p k / w p k$ Meckel Syndrome rat (left). Stereocilia should be in a $V$ configuration (noted) but the $V$ is distorted in many cells. Retinal photoreceptors (right) have cilia (arrows) which connect the inner and outer segments. Outer segments develop photosensitive discs (in normal retina, middle image) which is lacking in wpk/wpk rats (right). 